EPJ Web of Conferences 59, 04010 (2013)

DOI: $10.1051 /$ epjconf/20135904010

(C) Owned by the authors, published by EDP Sciences, 2013

\title{
Changes of implosion dynamics derived by difference of equation of state
}

\author{
Yu Komatsu ${ }^{1}$, Toru Sasaki ${ }^{1}$, Takashi Kikuchi ${ }^{1}$, Nob. Harada ${ }^{1}$ \\ and Hideo Nagatomo² \\ ${ }^{1}$ Nagaoka University of Technology, Kamitomioka 1603-1, Nagaoka, Niigata 940-2188, Japan \\ ${ }^{2}$ Institute of Laser Engineering, Osaka University, Yamadaoka 2-6, Suita, Osaka 565-0871, \\ Japan
}

\begin{abstract}
To evaluate an implosion dynamics with different equation-of-state (EOS) models in inertial confinement fusion (ICF), we compare two-dimensional radiation hydrodynamics simulation with QEOS, ideal gas EOS, and SESAME. The maximum density achieved during the implosion for the ideal gas EOS is higher than that for the QEOS. The sound velocity for the SESAME is faster than that for the QEOS. These results indicated that the EOS models affect the implosion dynamics in ICF.
\end{abstract}

\section{INTRODUCTION}

During an implosion process of inertial confinement fusion (ICF) [1], target materials passes through a wide regime in density and temperature. The temperature is changed from $10 \mathrm{~K}$ to $10^{8} \mathrm{~K}$, and the density is varied from that of solid state to 1000 times solid density. To generate the inertial fusion energy, we should clearly understand the hydrodynamics during the implosion process of fuel pellet in the wide density-temperature regime. Especially, the implosion dynamics is dominated by complex physical phenomena, such as strong shock wave, thermodynamics, atomic process, radiation transport, phase transition, and so on [2].

Recently, warm dense matter (WDM), which is a thermodynamic regime defined by 0.01-1 times solid density with temperature range of $0.1-10 \mathrm{eV}$, pointed out the difference of existing EOS models [3]. The WDM region includes the phase-transition from solid-state to plasma, degenerated electrons, and coupled ions. Hydrodynamic codes for the numerical analysis require the use of EOS models described by the thermodynamic functions of both electrons and ions. One of the models widely used in ICF is theoretical EOS model, such as the model based on the Thomas-Fermi theory for the electronic contribution to the EOS [4]. On the other hand, a tabular EOS model including theoretical approaches and experimental data is also used [4].

We investigate the important physics for EOS in the implosion of ICF by numerical simulations using hydrodynamic code with various EOS models. We will construct the essential and easy to use EOS model to consider the important phenomena for the implosion. To understand the effect on implosion dynamics with EOS model including the WDM state, we demonstrate to evaluate the changes of implosion dynamics due to the difference of EOS models.

\section{NUMERICAL SIMULATION AND EOS MODELS FOR ICF IMPLOSION}

To evaluate the implosion process, we used the two-dimensional radiation hydrodynamics simulation code, PINOCO [5]. In PINOCO, mass conservation, momentum, electron energy, ion energy, equation of states, laser ray trace, laser absorption, radiation transport, surface tracing, and other related equations

This is an Open Access article distributed under the terms of the Creative Commons Attribution License 2.0, which permits unrestricted use, distribution, and reproduction in any medium, provided the original work is properly cited. 
EPJ Web of Conferences
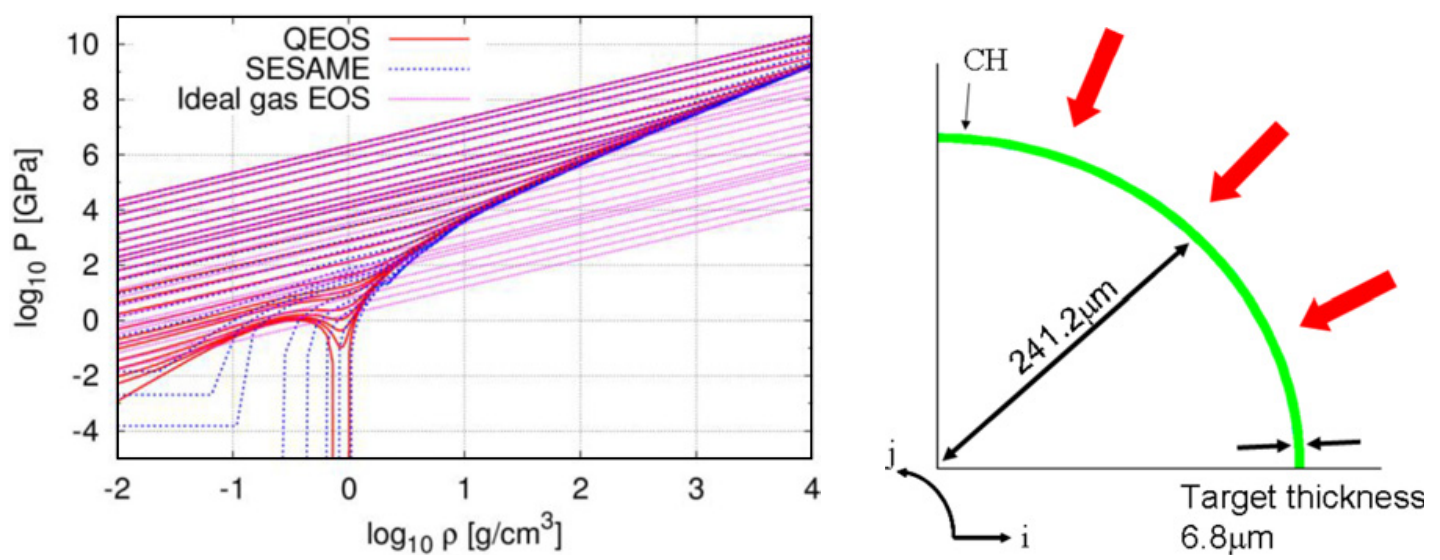

Figure 1. Comparison of QEOS, ideal gas EOS, and SESAME pressure curves of $\mathrm{CH}$ at constant

Figure 2. Target configuration. temperature.
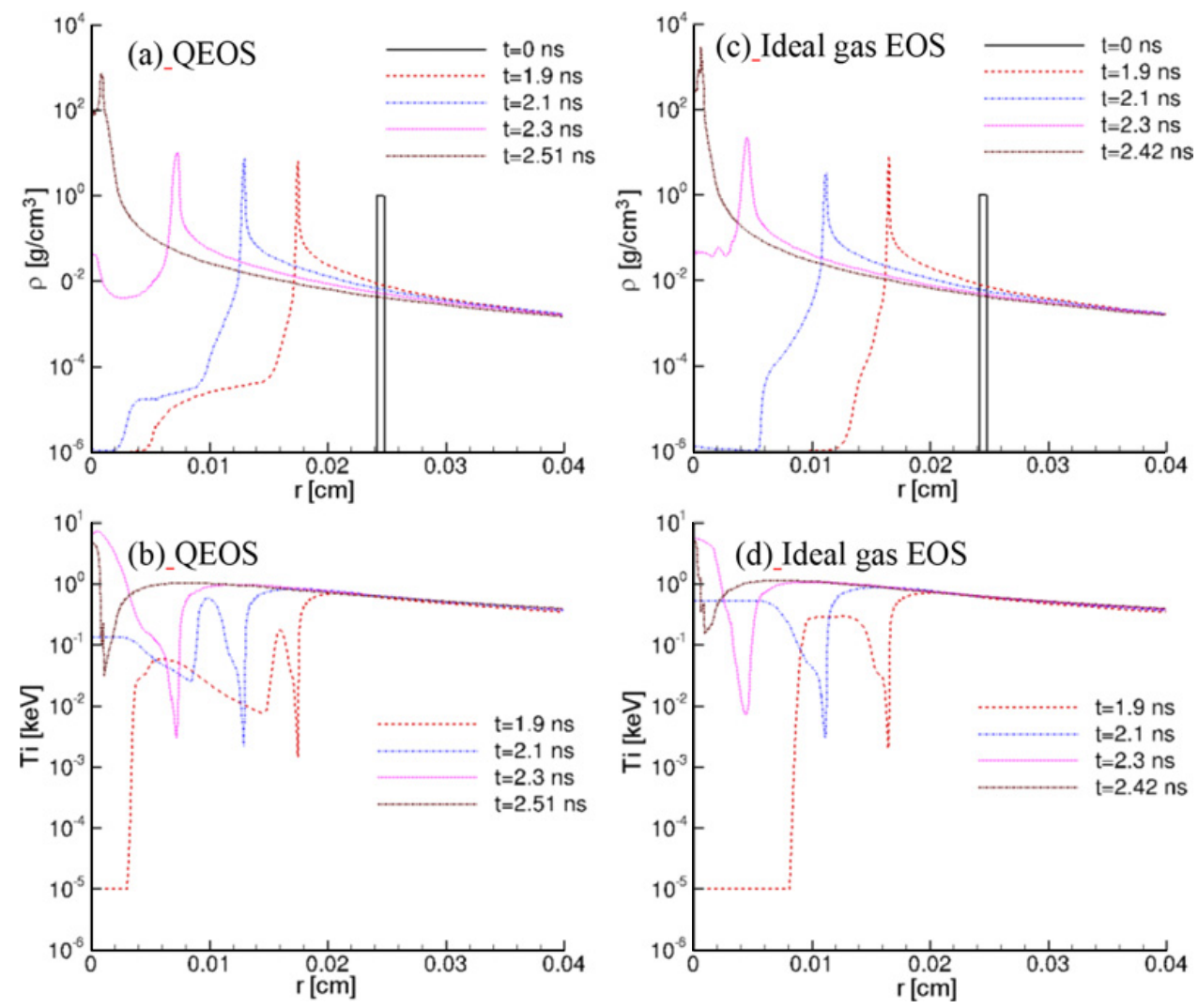

Figure 3. Time-evolution of radial profiles of the density and ion temperature, (a) and (b) for QEOS, (c) and (d) for ideal gas EOS. The solid line indicates the profiles at the initial condition $(t=0 \mathrm{~ns})$, and the dotted lines denote the profiles for $t=1.9 \mathrm{~ns}, 2.1 \mathrm{~ns}$, and $2.3 \mathrm{~ns}$, respectively. The profiles at $t=2.51 \mathrm{~ns}$ for QEOS and $t=2.42 \mathrm{~ns}$ for ideal gas EOS show the density and temperature at the maximum compression. 

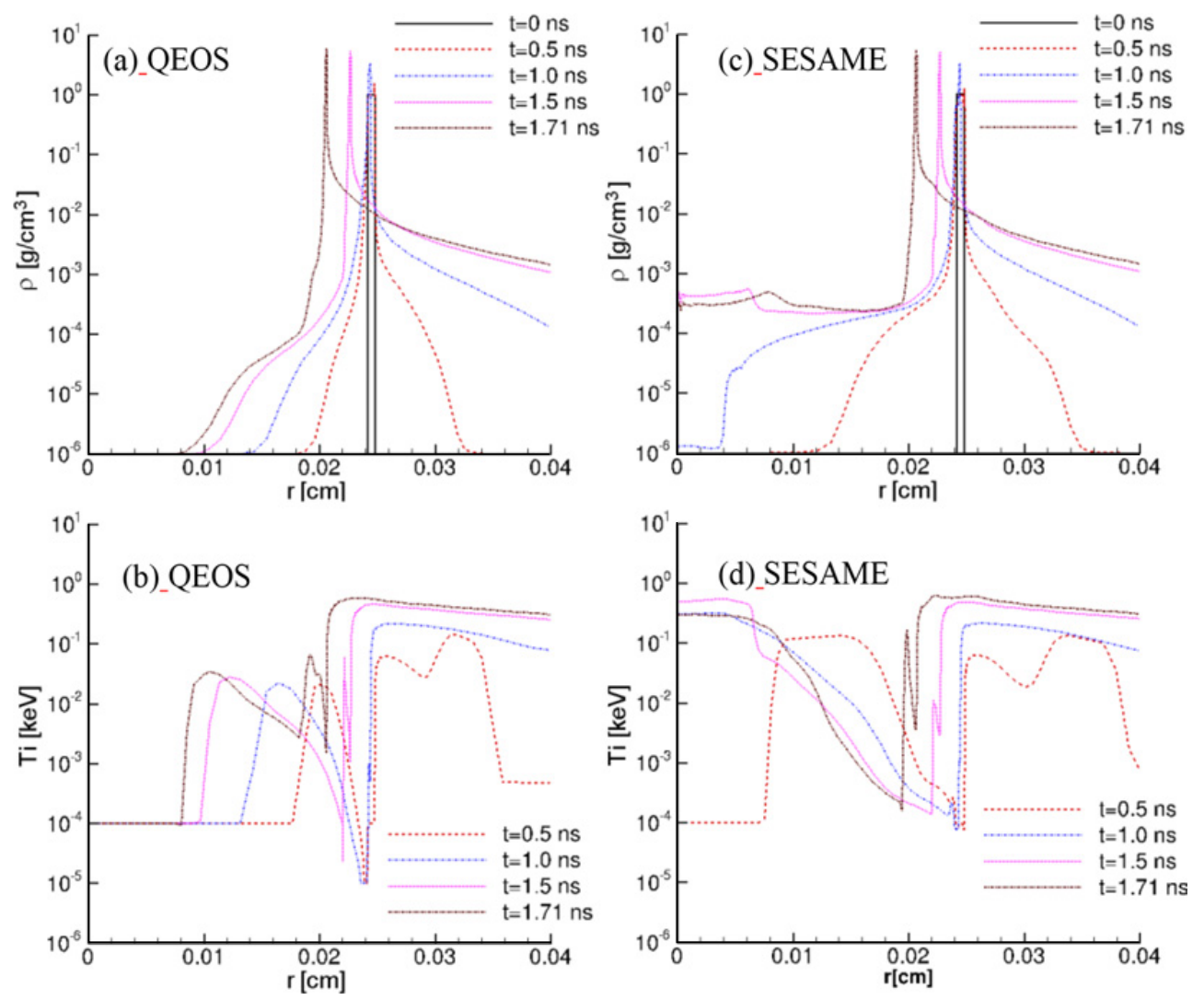

Figure 4. Time-evolution of radial profiles of the density and ion temperature, (a) and (b) for QEOS, (c) and (d) for SESAME. The solid line indicates the profiles at the initial condition $(t=0 \mathrm{~ns})$, and the dotted lines denote $t=0.5 \mathrm{~ns}, 1.0 \mathrm{~ns}, 1.5 \mathrm{~ns}$, and $1.71 \mathrm{~ns}$, respectively.

are solved simultaneously. The EOS implemented in PINOCO is based on the quotidian EOS (QEOS) [6] with a fitting formula [7].

The QEOS and SESAME [8] are usually used in numerical simulations for ICF. In QEOS, the cold and electron thermal property are based on semi-empirical bonding correction and the Thomas-Fermi model, respectively. The ion thermal properties are evaluated with Cowan's model. Thermodynamic derivatives such as specific heat, sound speed, can be easy to use from composed formulas.

The SESAME EOS are tabular data of the pressure and internal energy per unit mass as a function of temperature and density for various materials [8]. The pressure and internal energy of SESAME should be interpolation of the finite number of data. Therefore, the thermodynamic derivatives should be carefully computed due to discontinuity of the models.

Figure 1 shows the pressure curves of $\mathrm{CH}$ at each constant temperature. The pressure for ideal gas EOS is lower than that for the QEOS and SESAME in a high density and low temperature regime. There is a difference in low temperature and near the solid density regime even between QEOS and SESAME.

Figure 2 shows an overview of the target structure in the numerical analysis. Spherical implosion of $\mathrm{CH}$ shell target is simulated. The thickness and radius of the shell are $6.8 \mu \mathrm{m}$ and $241.2 \mu \mathrm{m}$. The background density and shell density are $10^{-6} \mathrm{~g} / \mathrm{cm}^{3}$ and $1 \mathrm{~g} / \mathrm{cm}^{3}$, respectively. To clarify the implosion dynamics from difference of EOS models, we neglect the radiation transport in this study. The uniform irradiation of laser in the target surface is assumed. The energy and pulse width of the laser are $2.0 \mathrm{~kJ}$ and 1.0 ns with Gaussian distribution, full width at half maximum (FWHM). 


\section{DENSITY AND TEMPERATURE PROFILES DURING IMPLOSION PROCESS}

Figure 3 shows the density and ion temperature profiles in radial direction obtained by the numerical simulation described in the previous section for QEOS and ideal gas EOS. The results indicated that the implosion dynamics of both EOS cases are different profile around the shock front. Thus, from these results in time evolution, the implosion velocity for the ideal gas EOS is faster than that for the QEOS. The difference of the density and temperature profiles is due to the degenerated pressure at over the solid density, which is $\log _{10} \rho \sim 0$ as shown in Fig. 1 .

The maximum density achieved with the ideal gas EOS is $3560 \mathrm{~g} / \mathrm{cm}^{3}$, and that with the QEOS is $741 \mathrm{~g} / \mathrm{cm}^{3}$. Therefore, the EOS models affect the achievable maximum density.

Figure 4 shows the density and ion temperature profiles in radial direction for QEOS and SESAME. In the interior of shell, the shock velocity for SESAME is faster than that for the QEOS. The compressibility $\kappa$ is expressed as follows,

$$
\kappa=\frac{1}{\rho}\left(\frac{\partial \rho}{\partial p}\right)_{T}=\frac{1}{\rho} \frac{1}{C_{s}^{2}},
$$

where $\rho$ is the density, $p$ is the pressure, and $C_{S}$ is the sound velocity expressed by

$$
C_{s}=\sqrt{\left(\frac{\partial p}{\partial \rho}\right)_{T}} .
$$

From Eq. (1), the difference of sound velocity causes the different compressibility, and affects the implosion velocity in the interior of shell. At $1.5 \mathrm{~ns}$ and $1.7 \mathrm{~ns}$, the ion temperature profile is different between both EOS models at the ablation front. The difference of pressure-density properties in the early time of the heating process may finally affect the implosion efficiency, because the fluid behaviour depends on the pressure history.

\section{CONCLUSIONS}

We investigated the implosion dynamics with the QEOS, ideal gas EOS, and SESAME by using PINOCO. The achieved maximum densities for QEOS and ideal gas EOS were different due to the degenerated pressure. The propagation profiles at the ablation front for QEOS and SESEAME were different. These results indicated that the EOS models affect the implosion dynamics in ICF. Particularly, the examination of the EOS model in the high density and low temperature regime is necessary because difference in pressure and speed of sound in the regime has an influence on implosion dynamics. We will construct useful EOS model for ICF in our future study.

This work was performed under the joint research project of the Institute of Laser Engineering, Osaka University).

\section{References}

[1] J. Nuckolls, et al., Nature 239, 139 (1972)

[2] S. Atzeni and J. Meyer-ter-vehn, Physics of Inertial Fusion (Oxford University Press, 2004)

[3] S.X. Hu, B. Militzer, V.N. Goncharov, S. Skupsky, Phys. Rev. Lett. 104, 235003 (2010)

[4] S. Eliezer, et al., Fundamentals of Equations of State (World Scientific, 2002)

[5] H. Nagatomo, et al., Phys. Plasmas 14, 056303 (2007)

[6] R. M. More, et. al., Phys. Fluids 31, 3059 (1988)

[7] K. Takami and H. Takabe, Technol. Rep. Osaka Univ. 40, 159 (1990)

[8] S. P. Lyon, J. D. Johnson, Group T-1, LA-CP-98-100 (1988) 